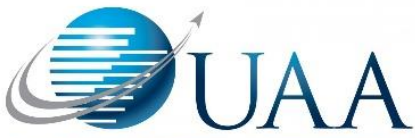

\title{
From Classroom to Industry: Human Factors in Aviation Maintenance Decision-Making
}

\author{
Bettina Mrusek \\ Embry-Riddle Aeronautical University \\ Stephanie Douglas \\ Embry-Riddle Aeronautical University
}

\begin{abstract}
The presence of human factors in aviation remains a critical area of research given the safety implications of human error. Understanding what specific factors contribute to human error allows managers and operators to take steps to mitigate these hazards. Several methods have been tested in the cockpit and cabin crew environments, but less attention has been given to the aviation maintenance sector, despite the prevalence of accidents resulting from human error. With the introduction of $\mathrm{AC}-172 \mathrm{~A}$, the FAA validated the need for additional research and training on the role of human factors in aviation maintenance errors. However, a key component in this process is often overlooked--the role of decision-making. In aviation maintenance, the environment can change rapidly. Technicians must react and adjust their behavior, and their decision-making abilities, accordingly. Human factors such as fatigue, pressure, and distractions can interrupt cognitive processes and judgment, and in turn, decision-making. As technicians adapt to these environmental challenges, strategies must be in place to facilitate optimal decisionmaking. Recommendations for addressing the presence of human factors in aviation maintenance and the resulting impact on the decision-making process include taking both a proactive and reactive approach to human error identification. Proactively screening for individuals who are too risk-averse or too comfortable with taking risks can help hiring managers employ the right personnel equipped to make appropriate decisions in high consequence industries, such as aviation. Additionally, by encouraging and reviewing hazard reports, steps can be taken to mitigate human error factors in the future. Anonymous hazard reporting tools such as the REPAIRER allow maintenance managers to leverage existing (and FAA-required) safety management systems (SMS) by including a human factors analysis.
\end{abstract}

\section{Recommended Citation:}

Mrusek, B. \& Douglas, S. (2020). From Classroom to Industry: Human Factors in Aviation Maintenance Decision-Making. Collegiate Aviation Review International, 38(2), (pending). Retrieved from http://ojs.library.okstate.edu/osu/index.php/CARI/article/view/8066/7433 
Recently, there has been a greater emphasis on the role of human error in aviation accidents, namely those that can be attributed to aviation maintenance errors (Rashid, Place, \& Braithwaite, 2013). While there are general guidelines that organizations can utilize to mitigate the presence of human error in these environments, less attention has been given to the unique challenges present in an aviation maintenance environment such as troubleshooting. This investigative process of determining the underlying cause of a maintenance failure can be timeconsuming and costly but is also accompanied by pressure constraints during aircraft on ground (AOG) scenarios or those in which there is a serious maintenance problem that is preventing the aircraft from flying. During these times, technicians can face incredible pressure to complete tasks as quickly as possible. To address potential safety hazards in aviation environments the FAA requires systematic procedures, practices, and policies, also known as Safety Management Systems (FAA, 2020). These programs support safety practices by viewing safety from a holistic perspective as opposed to merely a reactionary one. In practice, SMS systems provide an architecture for aviation organizations to proactively manage safety (Stolzer, Friend, Truong, Tuccio, \& Aguiar, 2018). In aviation maintenance environments, however, the decision-making process that occurs while troubleshooting AOG aircraft or other high-pressure scenarios has not been fully explored. While Aeronautical Decision-Making has proved effective in the cockpit (Harris \& Li, 2016), its application to the aviation maintenance environment could similarly prove useful especially when incorporated into training environments. This concept, combined with effective hazard reporting tools, could improve the effectiveness of FAA-mandated SMS programs in an aviation maintenance environment.

\section{Background}

Human error accounts for nearly $80 \%$ of all major Federal Aviation Regulation (FAR) Part 121 accidents (Marais \& Robichaud, 2012). Much research has been done to address this problem, with most efforts focused on the cabin and cockpit environments (Bienefeld \& Grote, 2014; Ford, Henderson, \& O'Hare, 2014; Peksatici, 2018). However, human error extends beyond the internal environment of the aircraft. Aviation maintenance crews also contribute to this percentage, although there is much less research to support this segment of the industry. While efforts have been made to improve the safety culture, a key component in this process is often overlooked; the decisions made during the troubleshooting process. Problem-solving in aviation maintenance is not strictly a linear process. In many cases, multiple decision points are considered throughout the troubleshooting process, which when coupled with the presence of one or more precursors to human error, can create a potentially unsafe environment (Rashid, Place, \& Braithwaite, 2013). The Dirty Dozen or the twelve most common precursors to human error in aviation maintenance (Dupont, 1993) is widely known and researched in aviation as well as other high-consequence industries (Marquardt, Treffenstadt, Gerstmeyer, \& Gades-Buettrich, 2015; Samad, Johari, \& Omar, 2018). However, the extent to which these occur throughout the troubleshooting process has not been fully explored. Given the lateral nature of the decisionmaking process and the livewire component of the aviation maintenance technician, strategies that minimize human error must be developed which account for variations in the decision- 
making process. Discounting these elements may conceal the root cause of the error. In this research paper, the authors will examine how the presence of human factors affects the decisionmaking process in aviation maintenance and provide recommendations for improving this process thus creating a more robust safety culture within the aviation maintenance environment.

\section{Human Error and the Aviation Maintenance Environment}

Aircraft accidents are an unfortunate reality in the aviation industry. While significant efforts have been made to increase safety at all levels of the industry since its inception, accidents still occur. As previously noted, human error accounts for nearly $80 \%$ of Federal Aviation Regulation (FAR) 121 Category aviation accidents, $10 \%$ of which can be attributed to aviation maintenance (Marais \& Robichaud, 2012). While this number may seem insignificant, as demand for air travel grows, the pool for which this percentage is derived will be much larger, validating the need to identify root causes and mitigation strategies for human error in aviation maintenance. While research has been conducted on this topic, they are often reactive, such as the National Transportation and Safety Board (NTSB) investigative reports. The NTSB performs official investigations on all aircraft accidents which has created a wealth of information researchers and industry personnel can use to modify or amend safety protocols. The information can be filtered by aircraft type, injury, or fatality numbers, as well as the underlying cause for the accident/incident (United States of Department Transportation, 1926). Over the last several years, the role of human error in accident causations has risen to the top (Marais \& Robichaud, 2012; Rashid et al., 2013). The distribution of this error has become an area of concern in the aviation industry, given its direct impact on overall safety. Empirical research studying aviation maintenance errors completed by Graeber and Marx (1994 noted four key categories of maintenance errors: omission (56\%), incorrect installation (30\%), wrong part (8\%), and other $(6 \%)$. Other studies yielded similar results regarding common causes of maintenance errors in aviation (Latorella \& Prabhu, 2000; Prabhu \& Drury, 1992).

Other research areas include the Dirty Dozen, Safety Management System programs, and Maintenance Human Factors, or MxHF (previously referred to as Maintenance Resource Management [MRM]). The Dirty Dozen, or the twelve most common precursors to human error in aviation maintenance, were identified by Gordon DuPont (1993) as a means of identifying preconditions that are most likely to result in human error. While there are certainly other conditions that could increase the likelihood of human error, these twelve were found to be the most common in aviation maintenance (Dupont, 1993). The original intent, which still stands today, was to bring awareness to how maintenance technicians contribute to accidents. The FAA along with the International Civil Aviation Organization (ICAO) has published guides as well as online training courses to support and educate aviation organizations in proactively identifying the twelve preconditions for unsafe acts (FAA Safety Team, n.d; ICAO, 1993).

A well-known proactive approach to managing safety in aviation is the Safety Management System (SMS) programs (Kearns \& Schermer, 2017; Stolzer, Friend, Truong, Tuccio, \& Aguiar, 2018). The FAA defines SMS as the "formal, top-down, organization-wide approach to managing safety risk and assuring the effectiveness of safety risk controls. It includes systematic procedures, practices, and policies for the management of safety risk" (FAA,

http://ojs.library.okstate.edu/osu/index.php/cari 
2020 pg A-2). SMS programs are comprised of four main components: safety policy, safety risk management, safety assurance, and safety promotion. Each area focuses on a different element of safety, promoting safety through organizational policies, proactively identifying risk hazards, then having programs to monitor and promote a safety culture. Combined, these elements form the foundation for a safety culture that proactively identifies and minimizes risk. However, only recently has the inclusion of human factors been included in SMS studies. Miller and Mrusek (2018) designed a hazard reporting system that fulfilled the FAA SMS requirements, while also accounting for the role of human factors, such as the Dirty Dozen in an aviation maintenance environment. As leaders in the aviation industry work to mitigate the hazards associated with human error, existing SMS programs present a viable opportunity given that they are already in place, and required by the FAA (FAA, 2020).

In 2017, the Federal Aviation Administration released an update to Advisory Circular (AC) 120-72, Maintenance Resource Management (MRM), which expanded traditional MRM concepts of effective communication and safety to include human factors (FAA, 2017). The new AC, appropriately titled Maintenance Human Factors Training, emphasizes training aimed at minimizing precursors to human error. While informative, the document serves as a guide only and is not as a mandatory requirement. On the surface, the inclusion of human factors training into existing safety management systems (SMS) would address the operational safety hazards found in an aviation maintenance environment.

Given that in 2012 10\% of all FAR 121 accidents were attributed to aviation maintenance errors (Marais \& Robichaud, 2012), it is clear that while the types of causes have been identified, the errors themselves remain today. This raises questions regarding the decision-making process that occurs before and following the improper maintenance actions. While aviation safety has improved substantially over the last several decades (Madsen, Dillon, \& Tinsley, 2016), some elements continue to pose hazards, such as the occurrence of aviation maintenance errors and the role of decision making in that process. If this element could be better understood, steps can be taken to ensure the right decisions can be made.

\section{The Decision-Making Process}

High consequence industries are those in which human life or quality of life is at stake. In industries such as aviation and health care, even routine decisions can be the difference between life and death. The decision-making process for those that work in these industries, therefore, is crucial. To improve the safety of these industries, regulatory agencies outline guidelines and provide resources to improve safety standards. In the U.S. aviation industry, the Federal Aviation Administration is responsible for setting such standards. While significant progress has been made in pilot training and aircraft equipment and systems, accidents still occur. The common factor among these accidents is human error (Latorella \& Prabhu, 2000). Despite advances in technology, the human element in aviation cannot be eliminated. Instead, a proactive approach to mitigating human error must be taken (FAA, 2017; Latorella \& Prabhu, 2000). Examining the decision-making process for personnel in high consequence industries, such as aviation, could uncover root causes in judgment errors. 
The concept of naturalistic decision-making (NDM) process is often utilized to replace the simplistic, sequential decision-making process. In contrast to decision paradigms that used pre-defined tasks given under controlled environment, NDM examines decisions made over time under natural conditions, relying on experience to cope with challenging decisions. NDM is most often applied to those occupations considered high consequence. In using a continuum of processes to adapt effectively to environmental constraints and the differing levels of ability among decision-makers, the NDM continuum ranges from an analytically based decision process to one more grounded in intuition (Klein, Oransanu, Calderwood, \& Zsambok, 1993). Various models have been developed in NDM from the Kleinst al. (1993) process-driven recognition primed model which describes how situational cues are used to identify patterns from previous experiences and then are used to construct patterns to make sense of the world around them. Pennington and Hastie (1986) decision model is an explanation based where the role of situation assessment and recognition will predict how decisions are made in situations in which there is incomplete information. Finally, Rasmussen's (1983) decision process model of typological cognitive control identifies different types of behavior to show the influence of individual values and goals in addition to habit and automaticity will influence decisions. The various models of NDM consistently identify decision making is not done or influenced by discrete isolated events or processes and situation assessment is essential.

Various factors contribute to behaviors in decision making. Understanding the context surrounding the decision is a key part of the process as is understanding how individuals function in their decision making. The emphasis in NDM is on the decision-maker to objectively assess situations versus the role of affect regulation on the decision-making process. Affect regulation is the ability of an individual to modulate their emotional state to adaptively meet the demands of their environment (Schore, 1994). Understanding how this influences decision-making is essential. In aviation maintenance, the environment can change rapidly. Personnel must react and adjust their behavior, and ultimately their decision-making abilities, accordingly. The Dirty Dozen identifies precursors to human error aviation that may stem from affect regulation. Fatigue, pressure, distractions, and stress are identified as emotional factors resulting in physical or mental tension. Such factors can interrupt cognitive processes and judgment pertinent to decision making (Chan \& Singhal, 2013; Lazarus, 1991).

\section{Aeronautical Decision Making}

Recognizing the need for a systematic approach to decision-making in aviation, the Federal Aviation Administration published an advisory circular (AC 60-22) on Aeronautical Decision Making (ADM) (1991). The FAA defines aeronautical decision making (ADM) as a "systematic approach to the mental process of evaluating a given set of circumstances and determining the best course of action" (FAA, 1991, pg 2). Noting the importance of risk assessment and stress management, the FAA, through the ADM process, acknowledges the role of personal attitudes in decision-making and how those attitudes can influence safety in the cockpit. The FAA also outlined a three-step model to assist pilots with incorporating the process; perceive, process, perform. The model is intended to be applied continuously, throughout the flight. Crew Resource Management (CRM) also evolved out of ADM, as a means of extending ADM beyond the cockpit and including all personnel involved in the flight process, such as crew members.

http://ojs.library.okstate.edu/osu/index.php/cari 
Despite its initial entrance into regulatory guidance by the FAA nearly thirty years ago, ADM remains a critical component in pilot training, as well as aviation ground operations. Research has shown that ADM can be improved by training (Harris \& Li, 2016;) but is also influenced by organizational culture and leadership (Valentin-Marian \& Venera, 2016). Additionally, despite regulatory support from the FAA in support of effective ADM training and practices, pilot fatigue has still been found to influence the decision-making process in the cockpit (Ballard, 2014). Since its inception, improvements and recommendations have been made on how best to implement effective ADM practices into the cockpit. However, this process has not been adequately researched or incorporated into aviation maintenance despite recent emphasis on the percentage of accidents due to human error within this environment. Approximately $80 \%$ of all major Federal Aviation Regulation (FAR) Part 121 aviation accidents can be attributed to human error, $10 \%$ of which are caused by aviation maintenance human error (Marais \& Robichaud, 2012). Given its proven track record in improving cockpit safety, ADM could provide an opportunity to improve safety in the aviation maintenance environment.

\section{From the Classroom to the Industry}

The best way to teach ADM is to introduce basic concepts of decision-making while putting individuals in situations where they can practice ADM with positive reinforcement for safe judgment and decision-making behaviors (FAA, 1991). Decision-making training must focus on real-world problem solving in which simulation training is an excellent environment for developing decision-making skills (Thomas, 2017). Simulation is recognized in high-risk industries such as aviation for decision-making training as it is very difficult to achieve effective training outside of the normal context decisions are made. In developing decision-making skills, the need is to focus on the use of simulation-based training in highly realistic work-based scenarios. Within high-risk industries, critical decision-making typically occurs in challenging, sub-optimal environments. To develop greater decision-making ability, individuals need exposure to such conditions to build resilience and continue to build non-technical skills. Simulation training provides a psychologically safe environment where effective training can take place (Thomas, 2017).

Simulation training is widely used in aviation, mainly in pilot training. The use of ADM training through simulation with pilots lead to decisions that are less rushed, ill-considered, and overall better decisions are made in terms of situation assessment and risk management ( $\mathrm{Li} \&$ Harris, 2008). Response times are also believed to shorten as further training and practice in ADM through simulation is completed. Within Li and Harris's (2008) study, pilots were found to demonstrate better performance as a result of training in ADM. After each simulator trial, the majority of the pilots who received ADM training applied the most appropriate ADM method for the given situation. In accidents where loss of control was cited as a factor, timely and effective decision-making that did not impede the pilot's cognitive capacity to fly the aircraft may have produced more favorable outcomes possibly avoiding accidents. Despite this sobering consideration, there is no specific requirement for decision-making to be taught in pilot training syllabi (Taylor, Dixon-Hardy \& Wright, 2014). The results of decision-making accidents are a product of incomplete data, lack of experience, or biases (Strauch, 2016). Decision-making can be improved by increasing situational awareness of all involved in decisions (Dekker, 2015). 
A simulation model that described the flight operations and maintenance of fighter aircraft during normal and conflict conditions in the Finnish Air Force was constructed to help maintenance designers in normal operating conditions allocate appropriate personnel and material resources. The ultimate goal of the simulation training model was to learn how to maximize the conflict time operational capability of the fleet. The model was a valuable aid in improving maintenance-related decision making and found useful for training purposes in classroom demonstrations and field training (Mattila, Virtanen \& Raivio, 2008). Overall, the evaluation of ADM training for pilots has found those that received ADM training outperformed those groups that did not. The trained groups had greater gains in risk management performance compared to those that did not receive the training ( $\mathrm{Li} \&$ Harris, 2007). Under conditions of time pressure, decision-makers draw on the training to help determine what is occurring in the current situation, suggesting that if decision-making is required in such circumstances, the practice of tasks under those conditions is necessary (Kaempf \& Orasanu, 1997). The simulations foster a better mental directory of scenarios a pilot can draw on in the stressful situations (Stokes \& Kite, 1999). A simple, short, cost-effective training program in the use of decision-making produces significant gains in decision-making performance (Li \& Harris, 2007). Such training can and should be integrated into current aviation maintenance training through simulation.

Skills acquired by simulation-based training adequately transfer to operative settings (Sturm et al., 2008) with firm scientific evidence of transfer from training in a virtual environment to real-world tasks (Rose et al., 2000). The intensive use of simulators has had a positive impact on commercial aviation safety and is accepted by operators, flight crews, unions, and regulators (Allerton, 2002). Simulation training offers practical training without incurring further risk. Incorporating scenarios through the simulation training allows training to be practiced over and over again providing a template form in which good decisions in various situations are constructed. The use of simulation in this manner is likely more effective than discussions in the classroom (Taylor et al., 2014).

In aviation and many other industries such as medicine and rail, it is understood that simulation training is a valuable teaching method for decision-making. In surveying aviation professors and instructors, the findings indicated the need for ADM instructional methods in flight training and that teaching ADM was not consistently used (Cassens, Young, Greenan, \& Brown, 2018). There were significant differences in how ADM instruction specific to good examples of decision-making and allowing students to make go/no go decisions for flights were incorporated into realistic training scenarios and lessons (Cassens et al., 2018). Scenario training was placed at the bottom of professors' and instructors' priorities indicating the efforts of the FAA encouraging scenario-based education have not been entirely successful (Cassens et al., 2018). The need to expose learners to various situations through simulation allowing them to develop into skilled decision-makers is not being fully optimized. While ADM training has been mostly focused on pilots, the need is also great in aviation maintenance. Few studies have attempted to develop a framework for maintenance optimization through decision-making (Alrabghi \& Tiwari, 2016). The need is for decision training within practitioners and academics that implements the simulation models with the available data and consideration of specific contexts (Van Horenbeek, Pintelon, \& Muchiri, 2010) for aviation maintenance. 
When teaching students about decision-making within complex systems, it is vital to incorporate methods and tools allowing learners to gain explicit and implicit knowledge about complexity and decision-making (Ku, MacDonald, Andersen, Andersen, \& Deegan, 2016). Such methods help learners identify where positive, meaningful outcomes can be made through interventions, directly make the interventions, and observe how their actions influence the system they are managing. Simulation-based learning increases intrinsic motivation in students, nurtures the ability to bring concepts and analytic skills together to solve a complex problem, and then change from linear to dynamic thinking (Ku et al., 2016).

\section{Discussion \& Conclusions}

Recent studies on decision-making training suggest specific training approaches may be most efficient when the decision-maker has had previous experience with similar situations, as is the case with the NDM process. Attempting to cover a breadth of aviation decision-making contexts in a generalized manner is less efficient and may be less effective when decisions are time-critical, as in an aviation maintenance environment. It is also important to emphasize context-based experiences in decision-making training, such as those used in the simulation. Aviation knowledge acquired through training and experience is essential for proper decision making. Aviation professionals must understand that successful decision making does not always involve the choice of the optimum solution. Given time pressures and uncertain conditions, success is making a choice that is sufficient to ensure safety.

In addition to training methods, a reporting system that proactively identifies human error using existing SMS programs would help to create awareness regarding the influence of human factors on aviation maintenance errors. Leveraging existing programs, such as SMS, minimizes the financial burden that often accompanies new procedures. The REPAIRER method appropriately addresses this need by including a human error component to hazard reporting, a method currently employed by aviation maintenance organizations. Leveraging the FAA's wellknown PEAR (people, environment, actions, resources) model for human factors, the REPAIRER expands this framework by including the rating and reporting of a hazard, as well as the execution and reevaluation of a mitigation strategy.

R-Report and Rate a human factors hazard
E-Environmental analysis
P-People involved
A-Actions of people identified
I-Investigate procedures
R-Resource evaluation
E-Execute recommended mitigation strategies
R-Reevaluate strategies and adjust

Another opportunity to manage human error in decision making would be during the hiring process. The development of a screening tool that measures risk tolerance would assist hiring managers by identifying those individuals that are too risk-averse or tolerant. High consequence industries require personnel with unique skill sets; those that can make appropriate 
decisions during times of intense pressure. Additional research is needed, however, to test these theories in an aviation maintenance environment. 


\section{References}

Allerton, D. J. (2002). The case for flight simulation in general aviation. Aeronautical Journal, 106, 607-612.

Alrabghi, A. \& Tiwari, A. ( 2016) A novel framework for simulation-based optimisation of maintenance systems. International Journal of Simulation Modeling, 15(1), 16-28.

Ballard, S. (2014). The U.S. commercial air tour industry: A review of aviation safety concerns. Aviation, Space, and Environmental Medicine, 85(2), 160-166. doi:10.3357/ASEM.3814.2014

Bienefeld, N., \& Grote, G. (2014). Shared leadership in multiteam systems: How cockpit and cabin crews lead each other to safety. Human Factors: The Journal of Human Factors and Ergonomics Society, 56(2), 270-286. doi:10.1177/0018720813488137

Cassens, R.E., Young, J.P., Greenan, J.P., \& Brown, J. (2018). Elements related to teaching pilots aeronautical decision making. The Collegiate Aviation Review International, 29(1)

Chan, M., \& Singhal, A. (2013). The emotional side of cognitive distraction: Implications for road safety. Accident Analysis \& Prevention, 50, 147-154.

Dekker, S.W.A. (2015). The danger of losing situation awareness. Cognition, Technology \& Work, 17, 159-161 (https://doi.org/10.1007/s10111-015-0320-8

Dupont, G. (1993) The Dirty Dozen Errors in Maintenance, Human Error in Aviation Maintenance (pp. 49-52). Federal Aviation Administration. Retrieved from: https://www.faa.gov/about/initiatives/maintenance_hf/library/documents/media/mx_faa_( formerly_hfskyway)/human_factors_issues/meeting_11/meeting11_7.0.pdf

Federal Aviation Administration (2020). Safety Management System order 8000.369C. https://www.faa.gov/documentLibrary/media/Order/Order_8000.369C.pdf

Federal Aviation Administration (2017). Advisory Circular 120-72A, Maintenance Human Factors Training. https://www.faa.gov/documentLibrary/media/Advisory_Circular/AC_120-72A.pdf

Federal Aviation Administration (1991). Advisory Circular 60-22, Aeronautical Decision Making. https://www.faa.gov/regulations_policies/advisory_circulars/index.cfm/go/ document.information/documentID/22624

Federal Aviation Administration Safety Team (n.d.). ALC-107: Dirty Dozen - Human error in aircraft maintenance training. Retrieved from: https://www.faasafety.gov/gslac/ALC/course_content.aspx $? \mathrm{pf}=1 \&$ preview=true \&cID $=10$ 7 
Ford, J., Henderson, R., \& O'Hare, D. (2014). The effects of crew resource management (CRM) training on flight attendants' safety attitudes. Journal of Safety Research, 48, 49-56. doi:10.1016/j.jsr.2013.11.003

Graeber, R. C., Marx, D. A. (1994). Human Error in Aircraft Maintenance, Aviation Psychology in Practice, 87-104.

Harris, D., \& Li, Wen-Chin (2016). Decision Making in Aviation.. Routledge.

International Civil Aviation Organization (1993). Circular 240-AN/144. Human Factors Digest No. 7, Investigation of Human Factors and Incidents. https://www.icao.int/isbn/Lists/Publications/DispForm.aspx?ID=308

Kaempf, G. L., \& Orsanu, J. (1997). Current and future applications of naturalistic decision making in aviation. In C.E. Zsambok \& G. Klein (Eds.), Naturalistic Decision Making (pp. 81-90). Lawrence Erlbaum Associates, Inc

Kearns, S. K., \& Aitken Schermer, J. (2017). Survey of attitudes toward aviation safety management system (SMS) training: Impact of gender and national culture. Aviation Psychology and Applied Human Factors, 7(1), 1-6. doi:10.1027/2192-0923/a000109

Klein, G. A.(1993). A Recognition-Primed Decision (RPD) Model of Rapid Decision Making. In G.A. Klein, J. Orasanu, R. Calderwood, C. E. Zsambok (Eds.), Decision Making in Action: Models and Methods. (pp. 138-147). Ablex Publishing Corporation.

Klein, G.A., Orasanu, J., Calderwood, R., \& Zsambok, C.E. (1993). Decision Making in Action: Models and Methods. Ablex Publishing Corporation.

Ku, M., MacDonald, R.H., Andersen, D.L., Andersen, D.F., \& Deegan, M. (2016). Using a simulation-based learning environment for teaching and learning about complexity in public policy decision making. Journal of Public Affairs Education, 22(1), 49-66.

Latorella, K. A., \& Prabhu, P. V. (2000). A review of human error in aviation maintenance and inspection. International Journal of Industrial Ergonomics, 26(2), 133-161. doi:10.1016/s0169-8141(99)00063-3

Lazarus, R.S. (1991). Emotion and Adaptation. Oxford University Press.

Li, W.C. \& Harris, D. (2008). The evaluation of the effect of a short aeronautical decisionmaking training program for military pilots. The International Journal of Aviation Psychology, 18(2), 135-152.

Madsen, P., Dillon, R. L., \& Tinsley, C. H. (2016). Airline safety improvement through experience with Near-Misses: A cautionary tale. Risk Analysis, 36(5), 1054-1066. doi:10.1111/risa.12503 
Marais, K. B., \& Robichaud, M. R. (2012). Analysis of trends in aviation maintenance risk: An empirical approach. Reliability Engineering \& System Safety, 106, 104-118. doi:10.1016/j.ress.2012.06.003

Marquardt, N., Treffenstadt, C., Gerstmeyer, K., \& Gades-Buettrich, R. (2015). Mental workload and cognitive performance in operating rooms. International Journal of Psychology Research, 10(2), 209.

Mattila, V., Virtanen, K., \& Raivio, T. (2008). Improving maintenance decision making in the Finnish Air Force through simulation. Interfaces, 38(3), 187-201, 222-223

Miller, M., \& Mrusek, B. (2018, July). The REPAIRER Reporting System for Integrating Human Factors into SMS in Aviation Maintenance. In International Conference on Applied Human Factors and Ergonomics (pp. 447-456). Springer, Cham.

Peksatici, O. (2018). Crew resource management (CRM) and cultural differences among cockpit crew - the case of turkey. Journal of Aviation/Aerospace Education and Research, 27(2), 1. doi:10.15394/jaaer.2018.1742

Pennington, N., \& Hastie, R. (1986). Evidence evaluation in complex decision making. Journal of Personality and Social Psychology, 51(2), 242-258. https://doi.org/10.1037/00223514.51.2.242

Prabhu, P., Drury, C.G. (1992). A framework for the design of the aircraft inspection information environment. In: Proceedings of the Seventh FAA Meeting on Human Factors Issues in Aircraft Maintenance and Inspection. Federal Aviation Administration, Washington, DC.

Rashid, H. S., Place, C. S., \& Braithwaite, G. R. (2013). Investigating the investigations: A retrospective study in the aviation maintenance error causation. Cognition, Technology \& Work, 15(2), 171-188. doi:10.1007/s10111-011-0210-7

Rasmussen, J. (1983). Skills, rules, and knowledge; signals, signs, and symbols, and other distinctions in human performance models. IEEE Transactions on Systems, Man, and Cybernetics, (3), 257-266.

Rose, F. D., Attree, E. A., Brooks, B. M., Parslow, D. M., Penn, P. R., \& Ambihaipahan, N. (2000). Training in virtual environments: transfer to real world tasks and equivalence to real task training. Ergonomics, 43(4), 494-511. https://doi.org/10.1080/001401300184378

Samad, A., Johari, M., \& Omar, S. (2018). Preventing human error at an approved training organization using Dirty Dozen. International Journal of Engineering and Technology, 7(4), 71-73. https://doi.org/10.14419/ijet.v7i4.13.21332

Schore, A. (1994). Affect regulation and the origin of the self. Routledge, New York, NY. 
Strauch, B. (2016). Decision errors and accidents: Applying naturalistic decision making to accident investigations. Journal of Cognitive Engineering and Decision Making, 10(3), 281-290.

Stokes, A., \& Kite, K. (1999). Grace under fire: The nature of stress and coping in general aviation. In D. O’Hare (Ed.), Human performance in general aviation (pp. 47-85). Aldershot, UK: Ashgate

Stolzer, A. J., Friend, M. A., Truong, D., Tuccio, W. A., \& Aguiar, M. (2018). Measuring and evaluating safety management system effectiveness using data envelopment analysis. Safety Science, 104, 55-69. doi:10.1016/j.ssci.2017.12.037

Sturm, L. P., Windsor, J. A., Cosman, P. H., Cregan, P., Hewett, P. J., \& Maddern, G. J. (2008). A systematic review of skills transfer after surgical simulation training. Annals of Surgery, 248(2), 166-179.

Taylor, A., Dixon-Hardy, D.W., \& Wright, S.J. (2014). Simulation training in U.K. general aviation: An undervalued aid to reducing loss of control accidents. The International Journal of Aviation Psychology, 24(2), 141-152.

Thomas, M. (2017). Training and assessing non-technical skills: A practical guide. CRC Press

United States Department of Transportation (1926). Air Commerce Act. Retrieved from: https://www.transportation.gov/content/air-commerce-act

Valentin-Marian I., \& Venera, C. (2016). Limitations of systemic accident analysis methods. INCAS Bulletin, 8(4), 167-174. doi:10.13111/2066-8201.2016.8.4.14

Van Horenbeek, A., Pintelon, L. \& Muchiri, P. (2010). Maintenance optimization models and criteria. International Journal of System Assurance Engineering and Management, 1, 189-200. https://doi.org/10.1007/s13198-011-0045-X 